\title{
Shape dynamics during deposit of simple metal clusters on rare gas matrices
}

\author{
P. M. Dinh ${ }^{1}$, F. Fehrer ${ }^{2}$, G. Bousquet ${ }^{1}$, P.-G. Reinhard ${ }^{2}$, and E. Suraud ${ }^{1}$ \\ ${ }^{1}$ Laboratoire de Physique Théorique, UMR5152, Université de Toulouse, \\ 118 Route de Narbonne, F-31062 Toulouse Cédex, France \\ ${ }^{2}$ Institut für Theoretische Physik, Universität Erlangen, Staudtstrasse 7, D-91058 Erlangen, Germany
}

\begin{abstract}
Using a combined quantum mechanical/classical method, we study the collisions of small $\mathrm{Na}$ clusters on large Ar clusters as a model for cluster deposit. We work out basic mechanisms by systematic variation of collision energy, system sizes, and orientations. The soft Ar material is found to serve as an extremely efficient shock absorber. The collisional energy is quickly transfered at first impact and the Na clusters are always captured by the Ar surface. The distribution of the collision energy into the Ar system proceeds very fast with velocity of sound. The relaxation of shapes goes at a slower pace using times of several ps. It produces a substantial rearrangement of the Ar system while the $\mathrm{Na}$ cluster remains rather robust.
\end{abstract}

PACS numbers: 36.40.Gk,36.40.Jn,36.40.Sx,36.40.Mr,61.46.Bc

\section{INTRODUCTION}

Clusters on surfaces have been much investigated during the past decade as can be seen, e.g., from the sequence of recent ISSPIC proceedings $1,2,3,4$. The topic remains of great interest, especially in relation to the synthesis of nano-structured surfaces. A possible way is here the direct deposition of size selected clusters on a substrate ${ }^{5,6}$. When deposited on a surface, a cluster undergoes a significant modification of its electronic structure and ionic geometry, because of the interface energy, the electronic band structure of the substrate, and the surface corrugation. These questions have been widely investigated from the structural point of view both at the experimental $7,8,9$ and theoretical 10,11,12,13,14,15,16,17 sides. The theoretical description of deposition dynamics is nevertheless a very demanding task. A fully microscopic approach treating all atoms and electrons in detail is extremely involved and applicable only to very small samples if at all. Most theoretical descriptions thus employ simple molecular dynamics approaches with effective atom-atom forces. There are, however, situations where a detailed description of electronic degrees of freedom is desirable, for example when metal clusters are involved where electronic shell effects are known to play a role in forming the structure.

A step forward is to combine a fully microscopical treatment of the cluster with a much simplified description of the surface. This is a valid and efficient compromise for inert substrates as, e.g., for the deposit of a metal cluster on an insulator surface. Such an approach was explored, e.g., in the case of $\mathrm{Na}$ clusters on $\mathrm{NaCl}$ in 17,18 for which Density Functional Theory was used for the Na electrons coupled to the surface via an effective interface potential, itself tuned to ab-initio calculations 13 . This approach, however, ignores any excitation and/or rearrangement of the surface itself, which supposes extremely inert materials. The next step is obviously to restore a minimum of surface degrees of freedom, still having electronically inert substrate in mind. Such a model was recently developed for the case of Na clusters embedded in rare gases $19,21,25$ where the cluster is again treated in full detail and the rare-gas atoms through classical dynamics of position and dipole polarization. Such a "hierarchical" approach is justified for electronically inert substrates. It has much in common with the coupled quantum-mechanical with molecular-mechanical method (QM/MM) often used in bio-chemistry ${ }^{22,23,24}$. The now explicit, although simple, handling of substrate atoms still restricts calculations to finite systems. The model developed in $20,21,25$, nevertheless, allows to explore a sufficiently large range of sizes to see the appearance of generic behaviors on the way towards the bulk.

The goal of this paper is to study the dynamics of deposition of a small $\mathrm{Na}$ cluster on a finite model of an Ar surface. To that end, we consider $\mathrm{Na}_{6}, \mathrm{Na}_{7}$, or $\mathrm{Na}_{8}$ clusters as projectile on $\operatorname{Ar}_{N}$ clusters of various sizes $(N$ between 7 and 87) as target. We analyze the kinetics of deposition in terms of the energy transfer between cluster and substrate, a point for which the explicit dynamical surface degrees of freedom are crucial. We shall also analyze the way in which the metal cluster adapts to the substrate and how the substrate gives way. This is done by tracking the evolution of the shapes of both partners during the deposition process and by varying Ar cluster size as well as initial kinetic energy. For this purpose we shall compare several small $\mathrm{Na}$ clusters with different shapes in the initial state. It should finally be noted that the considered setup (Na cluster on $\mathrm{Ar}_{N}$ ) has two aspects. First, it is an example of cluster-cluster collisions involving partners of very different size and nature, as such a topic of its own interest. Second, we have in mind an exploratory study of deposition on a Ar surface for which we consider large, but finite systems, a method which has been used also in experimental studies, see e.g. ${ }^{26}$. The widely used alternative is to simulate the infinite surface in terms of a small, but periodically copied, simulation box, see e.g. ${ }^{27}$. This better allows to include long range effects but limits the structural rearrangement of the surface. Thus modeling the surface by finite clusters is probably more appropriate for our intention to analyze the shape dynamics.

The paper is organized as follows. Section II provides

Typeset by REVTEX 
a short presentation of the model and of the systems considered in the test cases. In section III, we analyze the deposition dynamics in terms of trends and energies. In section [V] we discuss the shape dynamics in more detail.

\section{MODEL}

As stated above we use a hierarchical approach in which the metal cluster is treated in full microscopic detail while the "surface" is described at a classical level granting each atom mobility as a whole and a dynamical dipole response. This is justified by the clear hierarchy of electronic binding in metals vs. rare gases. The many different ingredients make a complete description of the model rather bulky. We give here a short account of the approach and refer to $\frac{19,21}{2}$ for a detailed layout.

The Na cluster is treated using quantum-mechanical single-particle wavefunctions $\left\{\varphi_{n}(\mathbf{r}, t), n=1, \ldots, N_{\mathrm{el}}\right\}$, for the valence electrons coupled non-adiabatically to classical molecular dynamics (MD) for the positions of the $\mathrm{Na}$ ions $\left\{\mathbf{R}_{I}, I=1, \ldots, N_{\mathrm{Na}}\right\}$. The electronic wavefunctions are propagated by time-dependent localdensity approximation (TDLDA). The electron-ion interaction in the cluster is described by soft, local pseudopotentials. This TDLDA-MD has been validated for linear and non linear dynamics of free metal clusters ${ }^{28,29}$.

Two classical degrees of freedom are associated with each Ar atom: center-of-mass $\left\{\mathbf{R}_{a}, a=1, \ldots, N_{\mathrm{Ar}}\right\}$, and electrical dipole moment $\left\{\mathbf{d}_{a}, a=1, \ldots, N_{\mathrm{Ar}}\right\}$. With the atomic dipoles, we explicitely treat the dynamical polarizability of the atoms through polarization potentials 30 . Smooth, Gaussian charge distributions are used for Ar ionic cores and electron clouds in order to regularize the singular dipole interaction. The $\mathrm{Na}^{+}$ions of the metal cluster interact with the Ar dipoles predominantly by their charge. The small dipole polarizability of the $\mathrm{Na}^{+}$ core is neglected. The cluster electrons do also couple naturally to the Coulomb field generated by the atoms.

The polarization potentials describe the long-range Coulomb part of the interactions. There remains to account for the short-range repulsion. The repulsive $\mathrm{Na}^{+}$Ar potential is taken from ${ }^{31}$. The pseudo-potential for the electron-Ar core repulsion has been modeled in the form of ${ }^{32}$, slightly readjusted by a final fine-tuning to the NaAr molecule (bond length, binding energy, and optical excitation). For the atom-atom interactions, we use a standard Lennard-Jones potential. A Van der Waals interaction is added, computed via the variance of dipole operators $19,21,32$. It provides a contribution to the longrange interaction which is crucial to produce the faint binding of the ground states for small Na-Ar systems. Its dynamical effects stay merely at a quantitative level.

We use standard methods 29 for the numerical solutions. We solve the (time-dependent) Kohn-Sham equations for the cluster electrons on a grid in coordinate space, using time-splitting for the time propa- gation and accelerated gradient iterations for the stationary solution. In the present calculations, we furthermore use the cylindrically-averaged pseudo-potential scheme (CAPS) as an approximation for the electronic wavefunctions $\mathrm{s}^{33.34}$. Both the $\mathrm{Na}$ ions as well as Ar cores and valence clouds are treated by classical molecular dynamics (MD) in full 3D. We have checked that a full 3D treatment of the electronic wavefunctions leads to similar results for the chosen systems which stay all close to axial symmetry.

\begin{tabular}{|l|l|l|l|}
\hline System & $\mathrm{Na}-\mathrm{Na}$ dimer & bulk $\mathrm{Ar}-\mathrm{Ar}$ & $\mathrm{Na}-\mathrm{Ar}$ dimer \\
\hline Bond energy & $800 \mathrm{meV}$ & $50 \mathrm{meV}$ & $5 \mathrm{meV}$ \\
\hline
\end{tabular}

TABLE I: Bond energies of dimers entering the calculations discussed in the text.

Table \ shows the binding energies for the three possible dimer combinations. There is a clear hierarchy of binding where the Na cluster is strongest bound, the Ar cluster one order of magnitude less bound, and the Na-Ar binding another order of magnitude smaller. This leads us to expect a fragile attachment of the two clusters to each other while the clusters as such remain intact with some readjustment of shape, particularly at the Ar site.

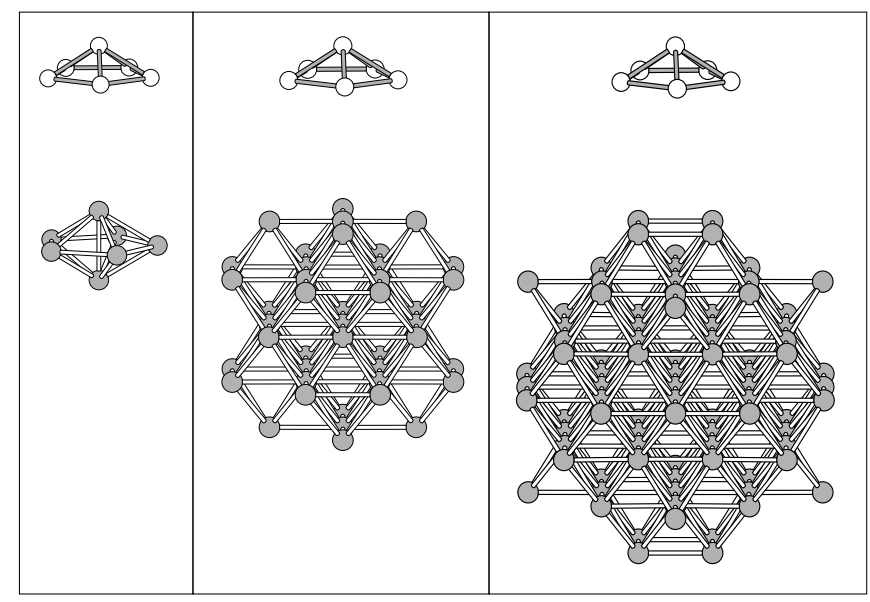

FIG. 1: Initial configurations for the deposition of $\mathrm{Na}_{6}$ (white balls) on $\mathrm{Ar}_{7}$ (left), $\mathrm{Ar}_{43}$ (middle), and $\mathrm{Ar}_{87}$ (right).

Three Ar clusters of different sizes are used as "substrate", $\mathrm{Ar}_{7}, \mathrm{Ar}_{43}$, and $\mathrm{Ar}_{87}$. The structures of these clusters are optimized for the given model by simulated annealing. The collision partners are various $\mathrm{Na}$ clusters taken in their ground state structure, again obtained by simulated annealing but this time coupled to static KohnSham iterations for the electrons. Most of the results presented below have been obtained for the strongly oblate $\mathrm{Na}_{6}$ cluster, composed of a ring of five ions and an outer ion, as can be seen from Fig. 1. Top configuration refers to the case where the pentagon hits first the Ar cluster. Reverse configuration corresponds to a $\mathrm{Na}_{6}$ up-side down. 
We also consider a few other clusters of comparable size but different shapes, especially $\mathrm{Na}_{7}$ and $\mathrm{Na}_{8}$. In $\mathrm{Na}_{7}$, the central pentagon is complemented by one top and, symmetrically, one bottom ion, which altogether delivers a much less oblate shape than $\mathrm{Na}_{6}$. The $\mathrm{Na}_{8}$ cluster, in turn, is built as two layers of four ions. Mind it is electronically close to sphericity because it contains the magic number of eight electrons. And indeed, the shape of free $\mathrm{Na}_{8}$ is, as far as it can be for a small finite cluster, close to spherical. In all cases, we take care of orienting the $\mathrm{Ar}_{N}$ relative to $\mathrm{Na}_{6}$ in such a way that it presents to the Na cluster the largest possible planar "surface". This can be seen in Fig. 11 where the initial configuration for the collision between $\mathrm{Na}_{6}$ and $\mathrm{Ar}_{7}, \mathrm{Ar}_{43}$ and $\mathrm{Ar}_{87}$ are displayed : The metal cluster initially faces respectively 1,5 , and $4 \mathrm{Ar}$ atoms. Note that in the largest system, the top $\mathrm{Na}$ ion starts above an interstitial position, whereas it is initially placed above an Ar atom in the two other cases.

The dynamics is started by giving the system a relative boost, with a "substrate" at $0 \mathrm{~K}$ temperature. We have studied the effect of "substrate" temperature up to $50 \mathrm{~K}$. No significant differences are observed what the deposition mechanisms and associated energy transfers are concerned, although the highest temperature represents a thermal energy of the same order of magnitude (about a factor 2-3 smaller) than the kinetic energy in Na. This shows that relevant effects are primarily of potential nature, especially the balance between short range (Ar core) repulsion and long range (Ar polarizability) attraction, both aspects well taken into account in our model. Note also that experimentally, deposition of metal clusters on rare gas substrates are performed at very low temperature (typically below $25 \mathrm{~K})^{35,36}$. Using just $0 \mathrm{~K}$ temperature is thus fully sufficient for the present aim of a qualitative study.

\section{ANALYSIS OF DEPOSITION MECHANISM}

\section{A. A visual example}

We first consider in Fig. 2 a typical deposition scenario for the example of $\mathrm{Na}_{6}$ on $\mathrm{Ar}_{43}$ with initial kinetic energy $E_{\text {kin } 0}=13.6 \mathrm{meV}$ per $\mathrm{Na}$ atom. The $\mathrm{Na}_{6}$ cluster is accelerated when coming closer to the Ar cluster, a long range polarization effect which is counterbalanced by the core repulsion only at very short distance. The pinning process proceeds stepwise with several bounces before the metal cluster is finally attached to the rare gas cluster. The overall shape of the Na cluster remains basically unchanged while the Ar cluster is somewhat rearranged, as was expected from the relative binding energies in Table II It should be noted that the initial kinetic energy per atom of the impinging cluster is smaller than the bonding energies of both $\mathrm{Na}_{2}$ and $\mathrm{Ar}_{2}$ dimers but about 2.5 larger than the binding energy of the $\mathrm{NaAr}$ dimer. Not surprisingly neither pure $\mathrm{Na}$ nor pure Ar bonds are broken, but in spite of the faintness of the NaAr bond one observes asymptotic stitching. This implies that an efficient kinetic energy transfer has taken place to cool down the Na-Ar interface, the excess kinetic energy being transferred to internal energy (potential and heat) of the Ar and/or Na clusters. A part of the potential energy is visibly used to reshape the Ar surface, in order to provide a more convenient contact plane for the $\mathrm{Na}_{6}$ cluster (see late times in the figure). The energy transfer mechanisms may a priori depend both on the initial kinetic energy and on the Ar cluster size. This will be analyzed in the coming sections.

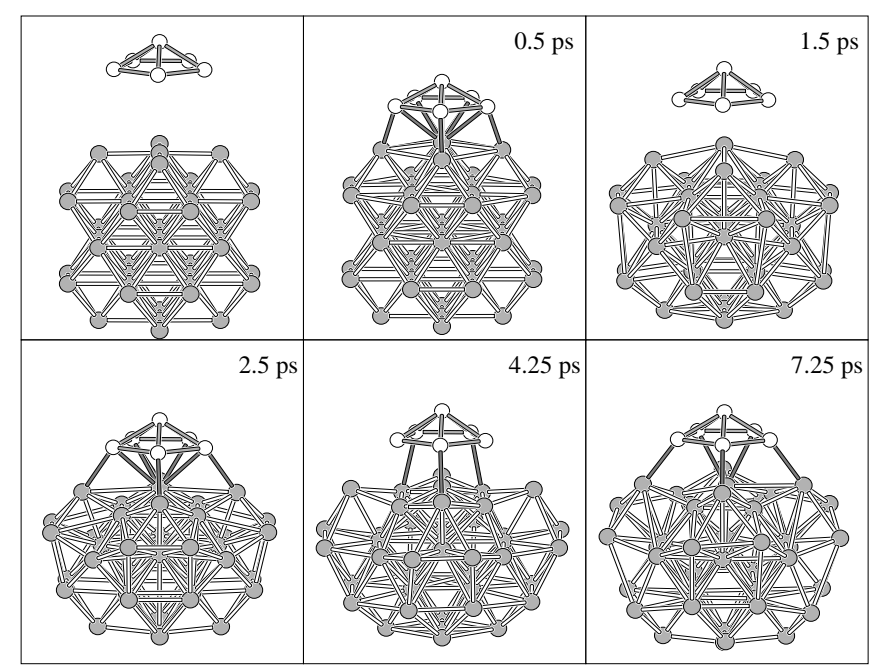

FIG. 2: Snapshots of the deposition of $\mathrm{Na}_{6}$ on a planar site of $\mathrm{Ar}_{43}$, with an initial kinetic energy of $13.6 \mathrm{meV} /$ ion. Time slots are indicated on each panel.

\section{B. Systematics on Ar cluster size}

We now turn to the question of the influence of the Ar cluster size and its heat capacity on the deposition process. Fig. 3 shows results for $\mathrm{Na}_{6}$ deposited on various Ar clusters all for the same initial kinetic energy of $E_{\text {kin } 0}=68 \mathrm{meV}$ per $\mathrm{Na}$ atom. The separation between the centers of mass of the two cluster is initially $30 a_{0}$ for all systems. The left panels show the detailed $z$ coordinates (symmetry axis and direction of collision) for $\mathrm{Na}$ ions and Ar atoms. It is obvious that, at this projectile energy, the $\mathrm{Ar}_{7}$ cluster is broken into pieces after the impact while both $\mathrm{Ar}_{43}$ and $\mathrm{Ar}_{87}$ are massive enough to resist the impact. Thereby the smaller $\mathrm{Ar}_{43}$ is visibly more perturbed (molten?) while the heaviest sample $\mathrm{Ar}_{87}$ even maintains its shell structure. The kinetic energies shown in the right panels explain that trend. Almost the same total kinetic energy of about $0.3 \mathrm{eV}$ is transferred in all three cases, independent from the $\operatorname{Ar}_{N}$ size. Distributing that equally over the Ar atoms associates a typical temperature to the Ar cluster of about $200 \mathrm{~K}$ for $\mathrm{Ar}_{7}$, 
$30 \mathrm{~K}$ for $\mathrm{Ar}_{43}$, and $10 \mathrm{~K}$ for $\mathrm{Ar}_{87}$, which fits perfectly to the observation of break-up, melting and stability. The largest sample thus serves as a reasonable model for deposit on a surface in this exploratory study. However true bulk is, of course, thermally very inert due to its large calorific capacity and one would expect even smaller values of temperature in the case of bulk deposition under the same kinematic conditions.

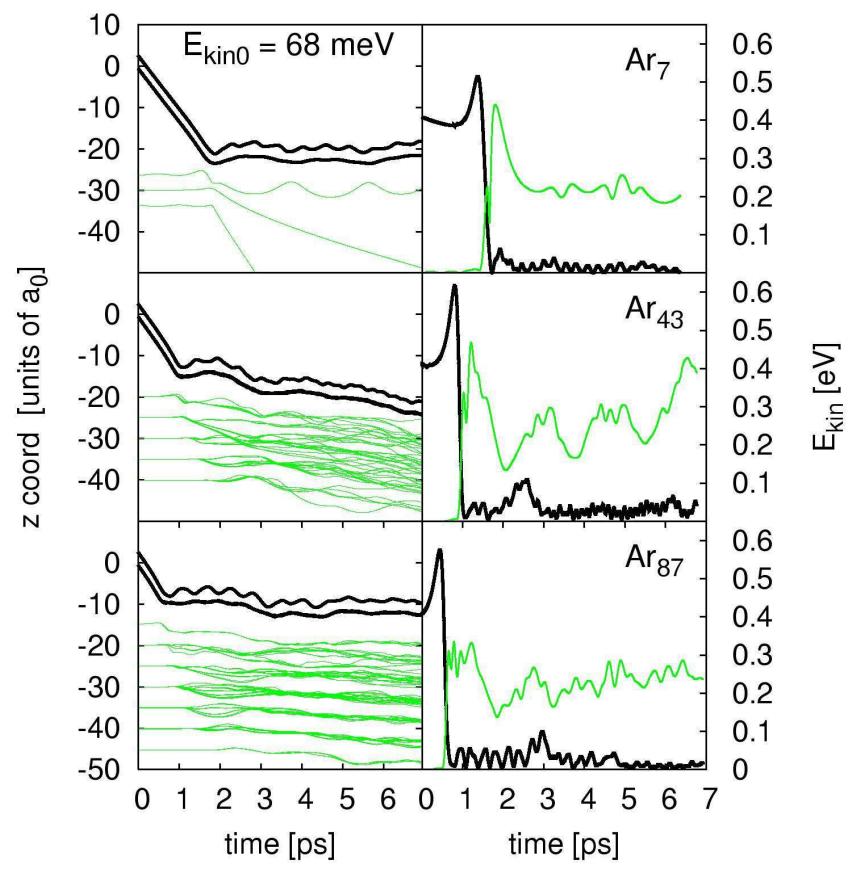

FIG. 3: Collision of $\mathrm{Na}_{6}$ (thick lines) with an initial kinetic energy of $68 \mathrm{meV} /$ ion, on $\operatorname{Ar}_{N}$ (thin curves) for $N=7$ (top), $N=43$ (middle) and $N=87$ (bottom). Left panels: $z$ coordinates; right panels: total kinetic energies, as a function of time.

A closer look to the kinetic energies in Fig. 3 shows a few interesting differences. The common feature is an initial energy transfer which leaves about half of the initial energy, i.e. around $0.3 \mathrm{eV}$, as kinetic energy in the Ar and very little kinetic energy for the $\mathrm{Na}$ cluster. The other half is invested in potential energy to provide the large spatial rearrangements. Differences are seen in further evolution. For $\mathrm{Ar}_{7}$ all rearrangement is finished after initial encounter and the kinetic energies remain unchanged later on. The severe perturbation of the medium sized $\mathrm{Ar}_{43}$ leads to ongoing rearrangements with long lasting exchange between potential and kinetic energy of the Ar cluster. As a side effect, there occurs also some re-heating of the $\mathrm{Na}_{6}$ cluster. Smaller reflow of potential into kinetic energy is seen for the heaviest $\operatorname{Ar}_{87}$.

Fig. 3 also spans very different time scales. The energy transfer at the moment of impact is surprisingly fast. The $\mathrm{Na}$ kinetic energy is fully given up within less than 0.5 ps and the energy distribution within the Ar cluster proceeds as a sound wave. This can be seen from the $z$ coordinates of the Ar atoms in the left panels below 2 ps. The perturbation propagates like a straight line through the Ar layers with a speed of $20-30 \mathrm{a}_{0} / \mathrm{ps}$. Long time scales, on the other hand, remain for the final relaxation processes. The successfully captured $\mathrm{Na}_{6}$ cluster continues to bounce and oscillate with a relaxation rate of order of $10 \mathrm{ps}$. The fine-tuning of the Ar cluster shape seems to proceed at about similar slow scale. Note, however, that this shape evolution initiated by the sound wave might differ for an infinite substrate where the sound wave would dissolve into deep layers. In the small finite systems here, the wave is dissipated by diffuse scattering from the opposite surfaces of the Ar cluster which turns collective energy into heat. A large part of the Ar kinetic energy seen in Fig. 3 thus becomes thermal energy. The case of largest Ar cluster, $\mathrm{Ar}_{87}$, behave somewhat different. It presents cleaner surfaces as compared to smaller clusters and it shows a faint reverberation of the wave when the latter reaches the edge of the cluster, as is observed in the lower left panel of Fig. 3. The reflected wave bounces back with almost the same velocity to the side which qas hit initially. There it returns some momentum to the $\mathrm{Na}_{6}$, as can be seen in the small revival of its kinetic energy at about 3 ps (lower right panel). This reverbation is, of course, due to the finite size of $\mathrm{Ar}_{87}$ and would not appear in the case of an infinite substrate. Nevertheless, from the metal cluster point of view, this reflection seems to change very little its final shape and distance to the Ar "surface". In that sense, this system still mimics the gross features of deposition on an infinite surface.

\section{Systematics on initial kinetic energy}

It is expected that the deposition depends on the velocity of the projectile. We analyze this effect in the case of $\mathrm{Na}_{6}$ deposition on $\mathrm{Ar}_{87}$, with varying $E_{\text {kino }}$. Results are plotted in Fig. \f for $E_{\text {kin } 0}$ between $6.8 \mathrm{meV}$ and $136 \mathrm{meV}$ per $\mathrm{Na}$ atom. The striking feature is the overall similarity of all the different cases. This concerns in particular the very fast and almost complete energy transfer from the $\mathrm{Na}$ kinetic energy to the other degrees of freedom. A common feature is also the quick capture of the Na cluster and its long standing residual oscillations. Of course details of the scenarios differ from one case to the next. The perturbation of the Ar cluster increases naturally with increasing initial energy. There is also an interesting effect at initial times. The kinetic energy of the $\mathrm{Na}$ cluster first increases before contact. That is due to the medium range polarization interaction which is attractive. The additional acceleration depends on the initial velocity. More energy is gained for the slower velocities because the cluster moves for a longer time in the attractive regime. Thus the kinetic energy is almost quadrupled for the lowest $E_{\text {kin } 0}=6.8 \mathrm{meV}$ while only $10 \%$ effect is left over for the fastest collision here. Besides these subtle differences in detail, the energy deposit proceeds in all 
cases the same way, namely in an extremely quick fashion leaving basically no residual kinetic energy at the side of the $\mathrm{Na}$ cluster projectile. One thus finds that in a relatively large range of projectile kinetic energies, the $\mathrm{Na}$ projectile is glued to the Ar target. Except again for oscillations in the most energetic case, the overall structure and position (with respect to the Ar target) of the deposited $\mathrm{Na}_{6}$ seems to depend very little on the initial energy.

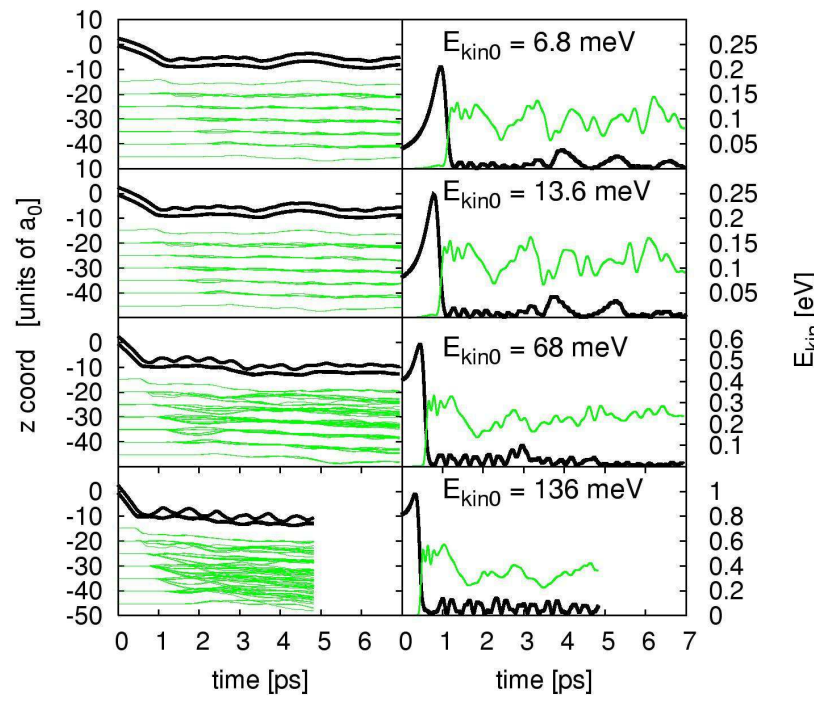

FIG. 4: $z$-coordinates (left) and total kinetic energies (right), as a function of time, in the collision of $\mathrm{Na}_{6}$ (thick curves) on $\mathrm{Ar}_{87}$ (thin curves), for four different initial kinetic energies $E_{\text {kin0. }}$.

\section{TIME EVOLUTION OF SHAPES}

\section{A. Cluster distance}

The above calculations show that the relaxation times of the whole deposition process goes beyond times we can reasonably simulate. However, we have seen from Figs. 3 and 4 that around 6-7 ps the kinetic energy of the Ar cluster and the cluster distance seems to reach a constant mean value. The closeness of the two clusters can be quantified in terms of a "deposit coordinate" $d$ that we define as

$$
d=\left[\frac{1}{N_{\mathrm{Na}} N_{\mathrm{Ar}}} \sum_{I, a} \frac{1}{\left|\mathbf{R}_{I}-\mathbf{R}_{a}\right|^{4}}\right]^{-1 / 4},
$$

where the index $I$ (respectively $a$ ) refers to the $\mathrm{Na}$ ion (respectively $\mathrm{Ar}$ atom) cores and $\mathbf{R}_{I}$ (respectively $\mathbf{R}_{a}$ ) to their position. The inverse puts weight on the closest partners. The actual power 4 in Eq.(1) is a matter of decision: we calculated with powers $n=1$ up to $n=$
7 and found that $n=4$ provides a good compromise between averaging and details.

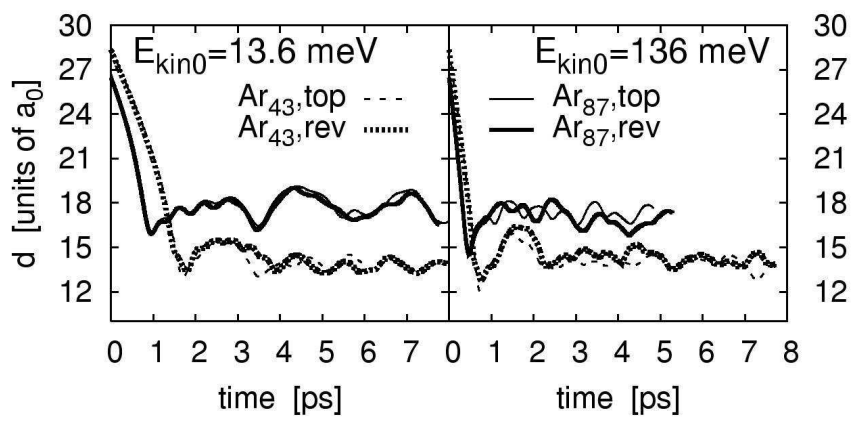

FIG. 5: Deposit coordinate $d$, defined in Eq.(1), as a function of time, for $\mathrm{Na}_{6} @ \mathrm{Ar}_{43}$ (dots and dashes) and $\mathrm{Na}_{6} @ \mathrm{Ar}_{87}$ (full lines), with $E_{\text {kino }}=13.6 \mathrm{meV} /$ ion (left panel) and 136 $\mathrm{meV} /$ ion (right panel). Results in both systems are presented for the top (dashes and thin curve) and the reverse (dots and thick curve) configuration of the $\mathrm{Na}_{6}$.

Fig. 5 shows the coordinate $d$ for two different sizes of the Ar clusters and for two different $E_{\text {kin0 }}$, i.e. initial $\mathrm{Na}$ kinetic energies per ion. The pattern are surprisingly similar for both energies and also similar for both sizes. The $\mathrm{Na}$ cluster is catched at first impact. There remain some oscillations in distance of about $2-3 \mathrm{a}_{0}$ which relax very slowly (order $10 \mathrm{ps}$ ). There is a global difference to the extend that the distance is closer for $\mathrm{Na}_{6} @ \mathrm{Ar}_{43}$. This is due to the larger rearrangements in that case which, in turn, provide a better contact area for the $\mathrm{Na}_{6}$.

We have also varied the orientation of the $\mathrm{Na}$ cluster. The standard configuration for $\mathrm{Na}_{6}$ was such that the ring of five $\mathrm{Na}$ ions stood closer to the surface while the top ion was pointing away (the situation denoted as "top" in the figure). Within axial symmetry, there is also the reverse situation where the top ion is facing towards the surface (denoted "rev" in the figure). The result for reverse initial configurations are also shown in Fig. 5. They are almost indistinguishable from the top configuration. This indicates that the $\mathrm{Na}_{6}$ remains basically unchanged in all cases. This will be corroborated in the next section studying shape dynamics.

\section{B. Global moments}

The results discussed up to now indicate that there may be substantial rearrangements at the side of the $\mathrm{Ar}$ substrate. Less is yet known about the shape of the $\mathrm{Na}$ cluster: do we have a soft landing, some plastic deformation or a wetting behavior? In order to quantify these questions we have performed a shape analysis in terms of the first three multipole moments both for $\mathrm{Na}_{6}$ and $\mathrm{Ar}_{N}$. These moments are given by

$$
\sqrt{\left\langle r^{2}\right\rangle}=\sqrt{\left\langle x^{2}+y^{2}+z^{2}\right\rangle}
$$




$$
\begin{aligned}
& =\left(\frac{1}{p} \sum_{i=1}^{p}\left(x_{i}{ }^{2}+y_{i}{ }^{2}+z_{i}{ }^{2}\right)\right)^{1 / 2}, \\
\beta_{2} & =\sqrt{\frac{\pi}{5}} \frac{1}{\left\langle r^{2}\right\rangle}\left\langle 2 z^{2}-x^{2}-y^{2}\right\rangle, \\
\beta_{3} & =\left(\frac{2}{5\left\langle r^{2}\right\rangle}\right)^{3 / 2}\left\langle z\left(z^{2}-\frac{3}{2}\left(x^{2}+y^{2}\right)\right)\right\rangle,
\end{aligned}
$$

where $p$ is either the number of $\mathrm{Na}$ atoms $N_{\mathrm{Na}}$ or the number of Ar atoms $N_{\mathrm{Ar}}$, and $x, y$ and $z$ are the coordinates of the $\mathrm{Na}(\mathrm{Ar})$ atom with respect to the center of mass of the $\mathrm{Na}(\mathrm{Ar})$ cluster. The r.m.s. radius $r$ stands for the overall extension (monopole moment) and the deformations are parameterized as dimensionless quantities which have immediate geometrical meaning independent of system size. For example, a value of $\left|\beta_{2}\right| \approx 0.8$ is a large quadrupole deformation with axis ratio of about $2: 1$.

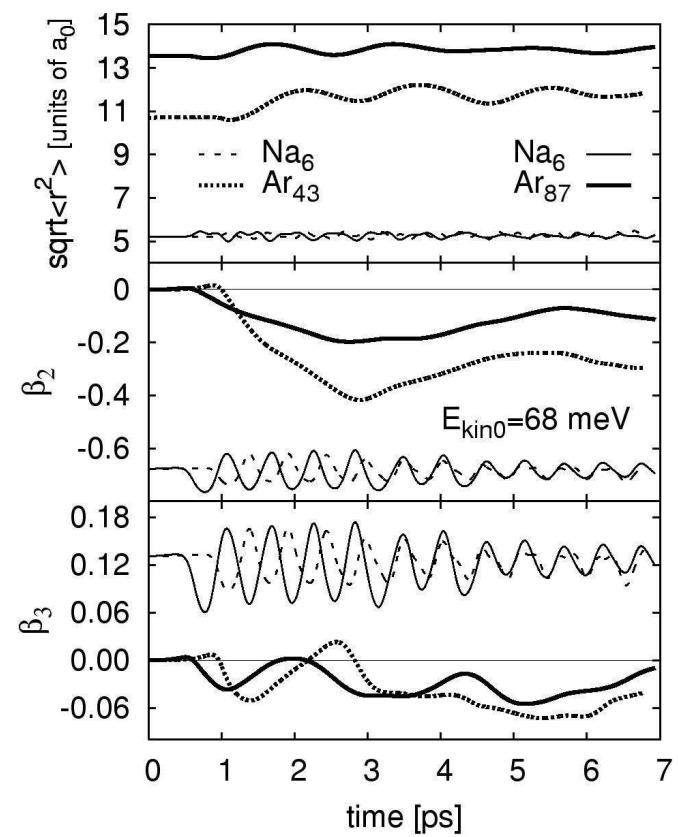

FIG. 6: Three first multipole moments $\sqrt{\left\langle r^{2}\right\rangle}, \beta_{2}$ and $\beta_{3}$, as a function of time, for $\mathrm{Na}_{6}$ (thin dashes and lines) deposited on $\mathrm{Ar}_{43}$ (thick dots) and $\mathrm{Ar}_{87}$ (thick full lines) for $E_{\text {kin0 }}=68$ $\mathrm{meV} /$ ion.

Fig. 6 shows the three momenta for the $\mathrm{Na}$ and $\mathrm{Ar}$ subsystem in the cases $\mathrm{Na}_{6} @ \mathrm{Ar}_{43}$ and $\mathrm{Na}_{6} @ \mathrm{Ar}_{87}$ for the moderate initial kinetic energy $E_{\text {kino }}=68 \mathrm{meV}$ per $\mathrm{Na}$ ion. The shape of $\mathrm{Na}_{6}$ is rather rigid in any case. There are some deformation oscillations short after impact which relax within about 3 ps. These oscillations are predominantly caused by the outer ion. The ring is tightly bound and stays more robust. Note that the relaxation is much faster than for the overall bouncing oscillations of the cluster (see Fig. 5). That is related to the binding properties shown in Table I that is, the
NaAr binding is softer. The Ar clusters, after the impact with $\mathrm{Na}_{6}$, increase slightly in size due to the heating by energy absorption. The growth is relatively larger for the smaller $\mathrm{Ar}_{43}$ which acquires a higher temperature as discussed above. The Ar clusters do also undergo a strong persistent change in deformation towards a sizeable oblate and somewhat pear-like shape. They obviously accommodate their configuration as to establish a most compact combined system.

For the case of $\mathrm{Na}_{6} @ \mathrm{Ar}_{43}$, the effect of initial kinetic energy and orientation (Na cluster in "top" or "rev" configuration, see section IVA are shown in Fig. (7) For the large $E_{\text {kino }}=680 \mathrm{meV} /$ ion (right panels), the $\mathrm{Ar}_{43}$ emits rapidly several atoms, thus yielding a rapidly increasing radius and octupole moment while a more moderate evolution emerges for the less violent $E_{\text {kin } 0}=136 \mathrm{meV} /$ ion. The collision with the $\mathrm{Na}_{6}$ top configuration is more violent because the whole ring bounces first and at once. This gives rise to an even higher Ar deformation for both initial energies. However, at the side of $\mathrm{Na}_{6}$, mean value and amplitude of the shape oscillations are similar in all cases, for both configurations and for both $E_{\text {kino }}$. Note the change of sign of $\beta_{3}$ for the reverse configuration. This indicates that the outer ion oscillates through the pentagon, within the given time once for $E_{\text {kino }}=136$ $\mathrm{meV}$ and twice at $680 \mathrm{meV}$. But the other two moments stay very robust in spite of the violence unload upon the $\mathrm{Ar}_{43}$ subsystem.

\section{Wetting behavior; comparison with $\mathrm{Na}_{7}$ and $\mathrm{Na} 8$}

We thus find that the $\mathrm{Na}_{6}$ is very robust under any conditions when deposited on the rather soft Ar material. It maintains its oblate shape and overall radius. The top ion is more loosely bound and may undergo larger oscillations which become apparent in the octupole momentum. The results for $\mathrm{Na}_{6}$ thus suggest that wetting of the surface is rather unlikely in that combination of materials. However, $\mathrm{Na}_{6}$ is not a very conclusive test case as it has already a close to planar structure. One ion still sticking out of the surface plane looks not so dramatic. In order to countercheck, we considered two neighboring clusters, namely $\mathrm{Na}_{7}$ (less oblate than $\mathrm{Na}_{6}$ ) and the almost spherical $\mathrm{Na}_{8}$. The results for the multipole moments are shown in Fig. 8. They are very similar to what we have seen already for collisions with $\mathrm{Na}_{6}$. The $\mathrm{Na}_{7}$ or $\mathrm{Na}_{8}$ clusters maintain basically their shape while the Ar basis undergoes a substantial rearrangement to create space for landing. There is not the slightest hint of a wetting. There are, of course, small shape oscillations in the $\mathrm{Na}$ clusters due to the internal excitation at the time of impact. It is interesting to note that these oscillations differ in detail between the two clusters. The $\mathrm{Na}_{8}$ with its magic electron number 8 seems to be more rigid and shows generally less oscillation amplitude. 


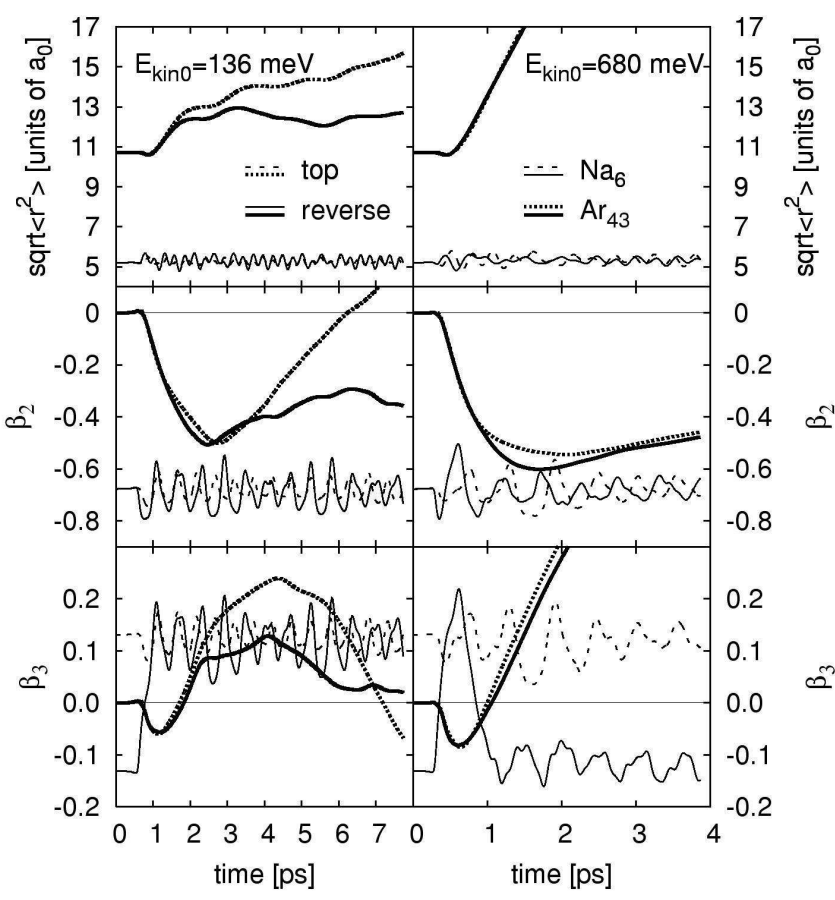

FIG. 7: Three first multipole moments, as a function of time, for $\mathrm{Na}_{6}$ (thin curves) deposited on $\mathrm{Ar}_{43}$ (thick curves), with $E_{\text {kino }}=136 \mathrm{meV} /$ ion (left) and $680 \mathrm{meV} /$ ion (right). On each panel are compared the moments obtained from the top (dots and dashes) and reverse (full lines) configurations.

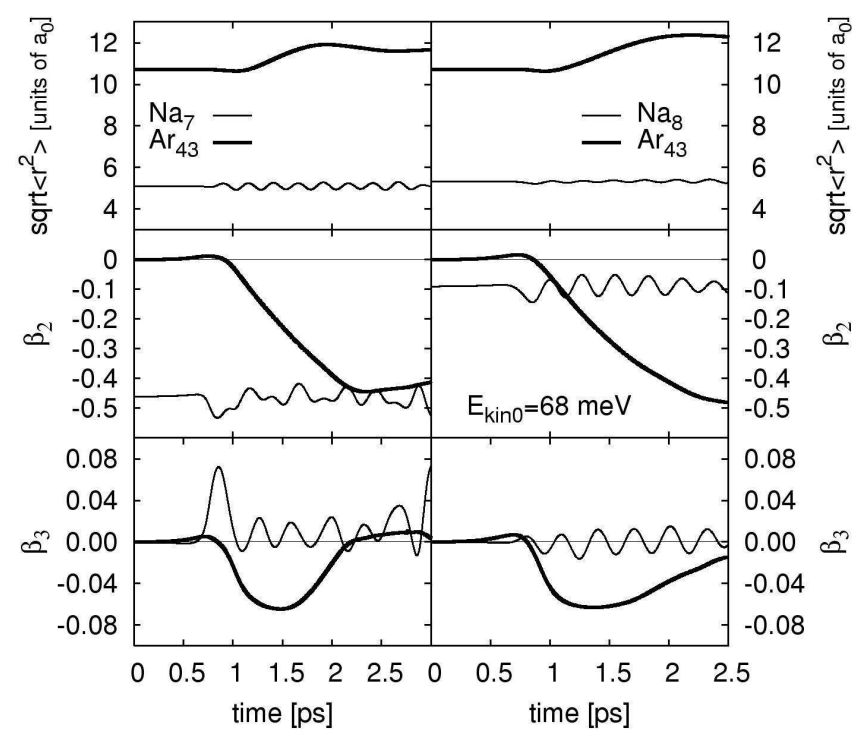

FIG. 8: The three multipole moments, as a function of time, for $\mathrm{Na}_{7}$ (thin curves, left panels) and $\mathrm{Na}_{8}$ (thin curves, right panel) deposited on $\mathrm{Ar}_{43}$ (thick curves), with $E_{\text {kino }}=68$ $\mathrm{meV} /$ ion.

\section{CONCLUSION}

In this paper we have investigated slow collisions of a small $\mathrm{Na}$ cluster with a large Ar cluster as a test case for deposit on an Ar surface. To that end, we have employed a hierarchical model treating the Na cluster in full detail by time-dependent density-functional theory and the $\mathrm{Ar}$ atoms at a thoroughly classical level with position and polarization as dynamical variables. We have studied in detail the influence of various parameters on the deposition scenario, namely initial collision energy, Ar cluster size, and relative orientation of the Na cluster.

We have found that, except for the case of the smallest Ar system, the basic scenario is robust in the sense that it depends very little on the collision parameters. The Na clusters are well bound while Ar binding is much softer and the Na-Ar binding even less. As a consequence, the Ar substrate constitutes a very efficient shock absorber. The impinging Na cluster is stopped immediately at first impact and attached to the Ar system while the latter takes over practically all initial Na energy. It seems impossible to tune conditions under which the $\mathrm{Na}$ cluster is reflected. Enhancing the initial energy rather leads to destruction of the Ar system. The initial large energy transfer from the $\mathrm{Na}$ cluster to the Ar system is very quick, taking less than 0.5 ps. The transferred energy is distributed also very quickly over all Ar atoms, propagating like a shock wave with speed of sound through the medium. About half of the energy goes into potential energy and part of it is used up for a large spatial rearrangement of the Ar cluster which aims to provide a most compact combined system. These rearrangements proceed on a slower time scale of 4-10 ps. At the side of the $\mathrm{Na}$ cluster, we have observed that in all cases its shape is little affected by the dynamical processes during deposit. It always safely attaches to the surface but only loosely bound and keeps nearly its free shape. The interface interaction is too weak to induce a wetting mechanism for this material combination.

Calculations with larger clusters and planar surfaces for the same combination confirm the above findings. It is now most interesting to check other combinations of materials. Work in that direction is in progress.

Acknowledgments: This work was supported by the DFG, project nr. RE 322/10-1, the french-german exchange program PROCOPE nr. 04670PG, the CNRS Programme "Matériaux" (CPR-ISMIR), Institut Universitaire de France, and the Humbodlt foundation, and has benefit from the Calmip (CAlcul en MIdi-Pyrnes) computational facilities. 
1 Proceedings of ISSPIC9, Lausanne 1998, Eur.Phys.J. D 9 (1999), Ed. U. Landmann

2 Proceedings of ISSPIC10, Atlanta 2000, Eur.Phys.J. D 16 (2001), Ed. I. Hertel

3 Proceedings of ISSPIC11, Strasbourg 2002, Eur.Phys.J. D 24 (2003), Eds. C. Bréchignac and Ph. Cahuzac

4 Proceedings of ISSPIC12, Nanjing 2004, Eur.Phys.J. D 34 (2005), Ed. G. Wang

5 C. Binns, Surf. Sci, Rep. 441 (2001).

6 W. Harbich, in "Metal Clusters at Surfaces, Structures", edts. K. H. Meiwes-Broer, Springer, Berlin, 2000.

7 Y.Z.Li, R.Reifenberger, R.P.Andres, Surf. Sci. 250, 1 (1991)

8 D.M. Schaefer, A. Patil, R.P. Andres, R. Reifenberger, Phys. Rev. B 515322 (1995)

9 Ch. Kuhrt, M. Harsdorff, Surf. Sci. 245, 252 (1995)

10 G. Rajagopal, R.N. Barnett, and U. Landman, Phys. Rev. Lett 67, 727 (1991)

11 H.P. Cheng, U. Landmann, Science 260, 1304 (1991)

12 H. Häkkinen, R.N. Barnett, U. Landmann, Europhys. Lett. 28, 263 (1994)

13 H. Häkkinen, M. Manninen, Europhys. Lett. 34, 177 (1996)

14 C. Kohl, P.-G. Reinhard, Z. Phys. D 3881 (1996)

15 C. Kohl, P.-G. Reinhard, Z. Phys. D 39225 (1997)

16 C. Kohl, E. Suraud, P.-G. Reinhard, Eur. Phys. J. D 11 $115(2000)$

17 A. Ipatov, E. Suraud, P.-G. Reinhard, Int. J. Mol. Sci. 4 (2003) 301

18 A. Ipatov, P. -G. Reinhard and E. Suraud, Euro. Phys. J D30, (2004) 65.
19 B. Gervais, E. Giglio, E. Jacquet, A. Ipatov, P.-G. Reinhard, and E. Suraud, J.Chem.Phys. 121, 8466 (2004).

${ }^{20}$ F. Fehrer, P. -G. Reinhard, E. Suraud, E. Giglio, B. Gervais, and A. Ipatov, Appl. Phys. A82, (2005) 151.

21 F.. Fehrer, M.. Mundt, P.-G. Reinhard, and E. Suraud, Ann. Phys. (Leipzig) 14, 411 (2005)

22 M. J Field, P. A. Bash, and M. Karplus, J. Comp. Chem. 11, 700 (1990)

23 J. Gao, Acc. Chem. Res. 29, 298 (1996)

24 N. Gresh and D. R. Garmer, J. Comp. Chem. 17, 1481 (1996)

25 F. Fehrer, P. -G. Reinhard and E. Suraud Appl. Phys. A 82, 145 (2005).

26 U. Buck and R. Krohne, Phys. Rev. Lett. 73, 947 (1994).

27 C. Xirouchaki and R.E. Palmer, Vacuum 66, 167 (2002).

28 P.-G. Reinhard and E. Suraud, Introduction to Cluster Dynamics, Wiley, New York, 2003.

29 F. Calvayrac, P.-G. Reinhard, E. Suraud, and C.A. Ullrich, Phys. Rep. 337, 493 (2000).

30 B G Dick and A W Overhauser, Phys. Rev. 112, 90 (1958).

31 G Reza Ahmadi, J Almlöf, and J Roegen, Chem. Phys. 199, 33 (1995).

32 F. Duplàe and F. Spiegelmann, J. Chem. Phys. 105, 1492 (1996).

33 B. Montag, P.-G. Reinhard, Z. Phys. D (1994).

34 B. Montag, P.-G. Reinhard, Z. Phys. D 33 (1995) 265.

35 J. T. Lau, W. Wurth, H.-U. Ehrke, and A. Achleitner, Low Temp. Phys. 29 (2003) 223.

${ }^{36}$ F. Conus, J. T. Lau, V. Rodrigues, and C. Félix, Rev. Sci. Instrum. 77 (2006) 113103. 